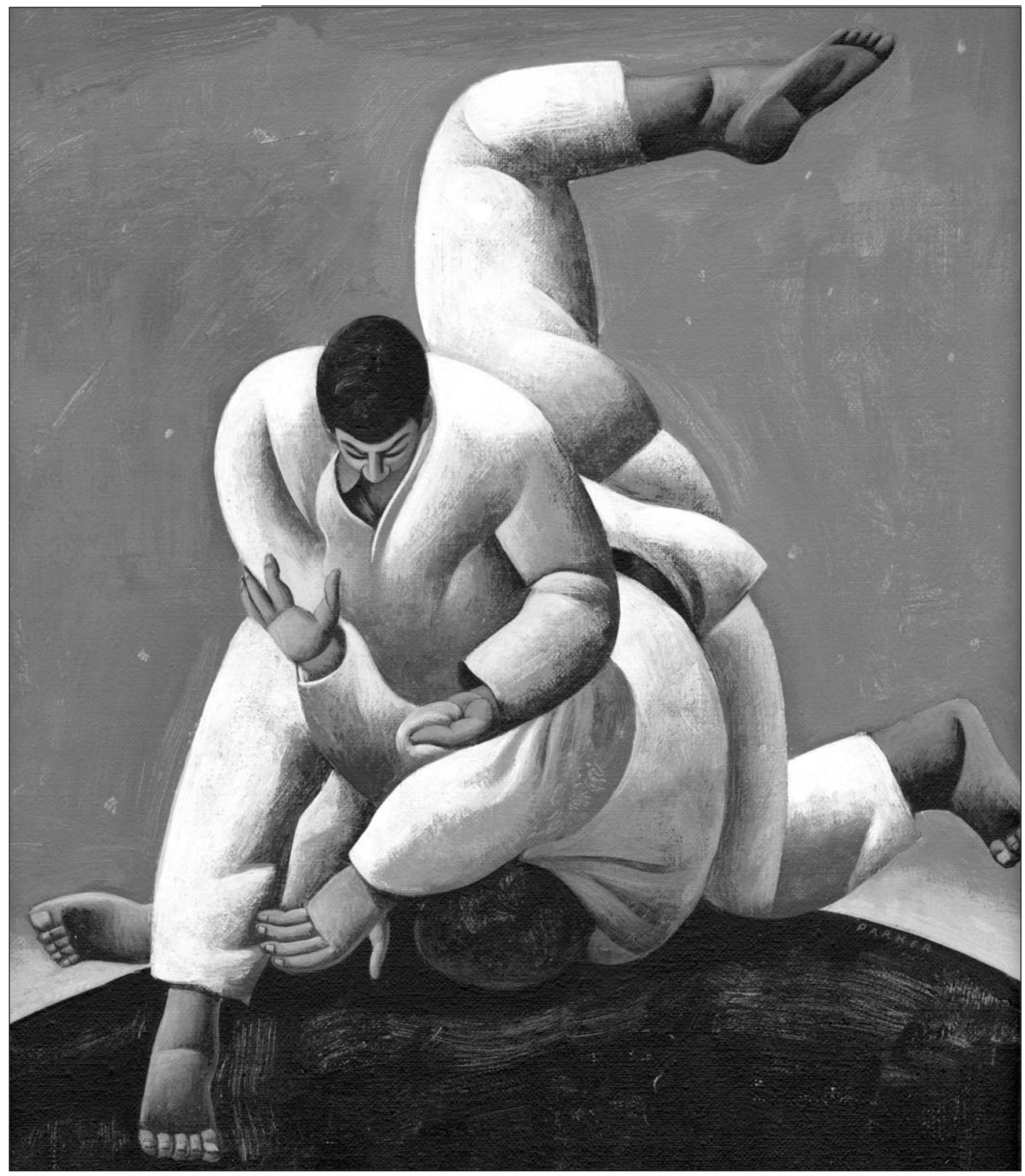

\title{
ARRIBA: Resumen
}

Pintura de Curtis Parker,

El presente artículo, "Forjando hombres sobre el tapiz", revisa las relaciones históricas entre la cultura popular estadounidense y las artes de combate. El artículo utiliza material de archivo de los s. XVIII a XX, CUYO ESTUDIO ESTÁ EN

TEMPE, AZ. TEL: (480) 820.6015 cparker@curtisparker.com www.curtisparker.com así como los análisis que realiza la literatura relevante en el ámbito de las ciencias sociales, para demostrar que las artes marciales son integrales a la sociedad estadounidense y que no son adquisiciones ad hoc de la cultura popular contemporánea. Entre las artes de combate que se discuten específicamente figuran prácticas como la esgrima, el combate con garrotes, la lucha, el boxeo a puño limpio y el judo.

Forjando hombres sobre el tapiz... $\diamond$ Geoffrey Wingard 


\section{FORJANDO HOMBRES SOBRE EL TAPIZ: Las “Artes Viriles" Tradicionales y las Artes Marciales Asiáticas EN los Estados Unidos}

POR GEOFFREY WINGARD

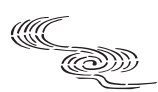

\section{Introducción}

En la era pos-Segunda Guerra Mundial, la mercantilización y diseminación de los deportes marciales basados en metodologías de combate asiáticas tradicionales ha llegado a ser un rasgo imperante en la cultura estadounidense. La institución y popularización de estas actividades marciales a todos los niveles de la sociedad -y la opinión prevalente de que son formas legítimas de recreación y de educación física y moral tanto para jóvenes como para adultos- se considera comúnmente como un ejemplo del desarrollo de una nueva institución en la sociedad estadounidense. Este fenómeno es tanto una consecuencia de la globalización cultural como un corolario de la apropiación americana de las tradiciones y culturas de pueblos que han sido ocupados y colonizados. Sin embargo, la adaptación de las artes marciales asiáticas a la sociedad estadounidense no es una ruptura con la tradición americana, ni tampoco es un ejemplo de desarrollo de una institución reciente en los EE.UU. Más bien, la popularización de las artes marciales y de los deportes de combate basados en anacrónicas metodologías de combate asiáticas debe verse como la continuación de un viejo proceso estadounidense consistente en adaptar diversas metodologías marciales tradicionales, a veces de elitistas, a la cultura popular americana. La apropiación y diseminación estadounidense de metodologías marciales de diversas naciones en diversos momentos, y la promoción de varias formas de recreación violenta, autoprotección y educación militarista del carácter es una tendencia que puede observarse no solamente hoy en día, sino a lo largo de toda la historia de los EE.UU.

A pesar de que el desarrollo de habilidades de combate prácticas ha sido, ciertamente, importante para los americanos por numerosas razones, la expansión de las oportunidades para practicar las artes marciales en América durante el último medio siglo parece no haber tenido precedentes. Como afirma el sociólogo Max Skidmore, "Difícilmente haya una sociedad de cualquier tamaño en Europa y en los países de habla inglesa en la que no sea posible recibir instrucción en una o más artes marciales" (Skidmore, 1995: 129).

Sin embargo, la práctica de todo tipo de estilos de combate, deportes y técnicas, tiene una larga historia en América. Las escuelas de esgrima italiana y francesa proliferaron ocasionalmente en las primeras áreas urbanas estadounidenses (Nadi, 1943: 22). La instrucción en la esgrima inglesa, y de un modo particular en la espada corta inglesa, estuvo presente en Norteamérica desde el periodo colonial al menos hasta finales del s. XVIII (Blackwell, 1734). Realmente, los métodos de combate americanos desarrollaron características únicas sobre la base de normas y prácticas regionales a lo largo de gran parte del s. XIX (Gorn, 1985: 18-43). La diferencia aparente entre la práctica tradicional de los ejercicios y rituales propios de las artes viriles, incluyendo la esgrima y otras habilidades de combate militaristas en la era posterior a la Segunda Guerra Mundial, y la práctica de las artes marciales asiáticas en la América de hoy en día parece basarse, en un examen cercano, en diferencias de atavíos, terminología y mitología, más que en cualquier otro tipo de diferencia significativa en relación a la accesibilidad a la instrucción o a la eficacia técnica. 
Detalle de la pintura de Curtis Parker.

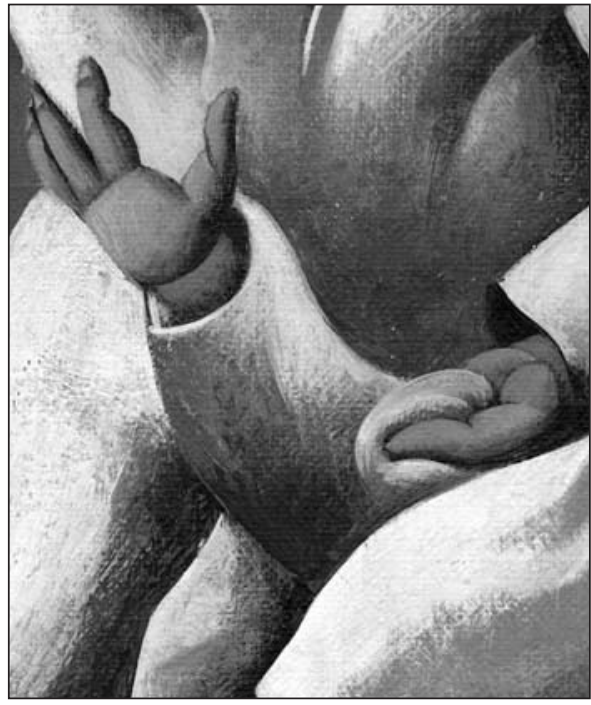

La diferencia, por tanto, es más de apariencia que de sustancia. El imaginario que rodea las artes marciales ha cambiado, pero no su sustancia y práctica en América. Este cambio en el imaginario ha ocurrido por una serie de razones y no es solamente, ni incluso principalmente, el resultado de la hegemonía estadounidense en el Pacífico tras la Segunda Guerra Mundial. De hecho, la apropiación y "americanización" de las artes marciales asiáticas comenzó bastante antes de que se produjese el conflicto americano-japonés en el Pacífico. Comenzó durante el primer periodo intensivo de interacciones entre OrienteOccidente a finales del s. XIX y principios del $\mathrm{XX}$. Este fue un periodo en el que la cultura pública occidental, y particularmente la cultura estadounidense, se afanaba en un intento consciente de modernización, aunque aún se basaba fuertemente en instituciones tradicionales. Sucedió en un momento en el que la élite estadounidense articuló un deseo consciente a favor del desarrollo industrial y de la necesidad de consolidar valores morales y sociales fuertes en jóvenes y adultos. Al mismo tiempo, los reformadores sociales de las clases altas pretendieron eliminar las prácticas y tradiciones de educación del carácter que consideraban vergonzantes y anacrónicas, mirando hacia el extranjero en busca de modalidades pedagógicas alternativas.

Un punto de encuentro educativo tradicional para el desarrollo del coraje, la fuerza y la lealtad en jóvenes y adultos americanos había sido a través de la práctica de las artes viriles, un compendio de ejercicios que incluían juegos en los que se sufría el riesgo de padecer un trauma físico o la muerte para fomentar entre los participantes el coraje y la lealtad personal hacia el grupo. Sin embargo, alrededor del cambio del s. XIX a XX, las artes viriles tradicionales, que incluían prácticas tales como la esgrima, el combate con garrotes, la lucha y el boxeo a puño limpio, habían dejado de contar con el favor del público, y la práctica de los nuevos deportes modernos aún tenía que llenar el vacío dejado por éstas. Los deportes modernos eran un nuevo tipo de institución social, un complejo de comportamientos y actitudes que complementaban y eran complementados por el industrialismo en América puesto que se basaban en temas y prácticas que se habían hecho populares en los juegos pre-modernos. A finales del s. XIX y principios del s. XX, los deportes amateur y profesionales, en oposición a los juegos participativos, aún tenían que lograr su aceptación universal ${ }^{1}$. En aquellos tiempos, los proselitistas de los deportes occidentales, los cristianos musculares y los defensores de la cultura física, miraron hacia el exterior en busca de prácticas que considerasen que pudieran integrarse en el medio social masculino occidental, y adaptarse para rellenar el hueco dejado tras el rechazo de las artes viriles tradicionales por parte de muchas de las élites ( $y$, consiguientemente, por parte del público). Encontraron, desarrollaron y adaptaron diversas prácticas marciales de todo el mundo para satisfacer sus necesidades, incluyendo de modo destacado la nueva, "científica" arte marcial importada de Japón (y parcialmente a través de Inglaterra) conocida como judo. Rápidamente adoptado por los aficionados victorianos y los orientalistas, el judo llegó a ser posteriormente el primero de una serie de deportes marciales asiáticos modernizados y occidentalizados que disfrutó de una amplia popularidad en Occidente ${ }^{2}$.

El estudio de la apropiación y diseminación del judo en América alrededor del cambio de siglo revela mucho sobre los acontecimientos sociales y culturales que sucedieron por todo el país en aquella época. Se ha señalado que "el modo en que combaten los hombres -quién participa, quién observa, qué reglas se siguen, qué está en juego, qué tácticas se permiten- revela mucho sobre las culturas y sociedades pasadas" (Gorn, 1985: 18). El estudio de los deportes en general y de las prácticas físicas que, como muchas

Forjando hombres sobre el tapiz... $\diamond$ Geoffrey Wingard 
artes marciales y particularmente en el judo, contienen aspectos tanto del enfrentamiento masculino tradicional como del deporte moderno (a pesar de que sus participantes evitan conscientemente la mayoría de tipos de competición profesional), puede decir mucho sobre las creencias e ideales de los participantes y de los observadores. Puesto que el deporte moderno, tal y como lo definió el historiador del deporte Allen Guttmann, sólo puede existir cuando hay participación y observación o patrocinio, el estudio de los deportes modernos implica el estudio de las personas a través de todo el espectro social (Guttmann, 1978). El estudio del deporte no es solamente el estudio de los participantes, frecuentemente de clases bajas o pobres, o de los habitualmente ricos patronos o de los aficionados y observadores de clases medias y trabajadoras, es el estudio de todos estos grupos y, lo que es más importante, es el análisis de sus interacciones. Debido a la fecha relativamente temprana de su introducción en Occidente, y puesto que es un sistema de combate que fue modelado intencionalmente para ajustarse a los requerimientos de un deporte moderno desde su concepción, el judo es particularmente útil como objeto de estudio (Carr, 1993: 169). El estudio de la introducción y popularización del judo en América puede, por tanto, arrojar luz sobre muchos temas que preocupan a los historiadores sociales, particularmente a aquellos interesados en los complejos conjuntos de normas y comportamientos que se sitúan en torno a la violencia, al control social y la perpetuación de la educación militarista en la sociedad estadounidense.

\section{Las “Artes Viriles" en los EE.UU.}

Previamente a la introducción del judo en los Estados Unidos a finales del s. XIX, las prácticas recreativas extenuantes y frecuentemente violentas eran incluidas en la categoría de prácticas atléticas popularmente conocidas como "artes viriles". Las artes viriles tradicionales en los EE.UU. incluían diversas prácticas públicas y privadas y juegos que implicaban el cultivo de la fuerza y del espíritu. Las artes viriles, tal y como las entendían los participantes desde finales del s. XVIII hasta principios del s. XX, incluían el boxeo, la lucha, la esgrima, el combate con palo, bastón y garrote, la gimnástica y los ejercicios calisténicos, derivados de, o usados para, intensificar los ejercicios militares. Las artes viriles, "las artes de combate de finales del s. XVIII hasta las primeras décadas del pasado siglo" (Wolf, 2000: 1), estaban ampliamente difundidas en América tanto entre las élites como entre los miembros de la clase obrera que buscaban fortalecer sus cuerpos, competir por premios y prestigio y conectar emocionalmente con un arquetipo anglosajón glorificado y viril, aunque en gran medida mitológico.

Antes del ascenso del movimiento del deporte profesional a finales del s. XIX y principios del s. XX, existía una muchísimo menor codificación de los deportes y juegos de la que existe hoy en día, y existe una particular escasez de registros sobre los toscos juegos practicados por el pueblo como recreación, derivados del trabajo manual, agrícola e industrial. Sin embargo, estos tipos de pasatiempos existieron, y mucha gente participaba en ellos como patrocinadores, observadores o jugadores. Puesto que es imposible determinar el número real de participantes, hechos como la diversidad de competiciones y prácticas, y la variedad y complejos conjuntos de reglas y normas aplicadas a las formas de recreación combativa anteriormente a la llegada del movimiento deportivo organizado a finales del s. XIX, hablan de la popularidad de las artes viriles para los componentes de diversas clases sociales, religiones, etnias y entornos sociales por todos los Estados Unidos.

A pesar de que pueda parecer absurdo a los observadores del s. XXI que la práctica de formas violentas de recreación pudiese ser vista como útil por cualquier otro motivo que no fuese la preparación militar o el entretenimiento popular, en los s. XVIII y XIX el cultivo de las habilidades marciales era visto como parte de la educación básica de todos los caballeros. En América, donde prevalecía una atmósfera de igualitarismo (al menos entre un segmento de los fieles republicanos), la idea de que la práctica de la violencia ritualizada tenía valor pasó rápidamente de manos de la élite al dominio público. Las artes viriles y las recreaciones marciales llegaron a ser populares, públicas y comerciales.

Revista de Artes Marciales Asiáticas \ Volumen 4 Número 2 (8-21) - 2009 
Este proceso ya había empezado a principios del s. XVIII. En 1734, Edward Blackwell, un inmigrante inglés a las colonias americanas, publicó un tratado sobre esgrima inglesa con espada corta. En Inglaterra, la esgrima con espada corta había sido atributo de los caballeros. La espada corta se había desarrollado como un arma para oficiales militares y caballeros que estaban lejos de la primera línea de fuego; era un arma para la defensa personal en situaciones donde no se tenía a mano un sable o un arma de fuego. En América, sin embargo, la esgrima con espada corta no era únicamente una práctica elitista (aunque las élites ciertamente patrocinaron a maestros de esgrima durante el s. XVIII), pero rápidamente estuvo disponible para el público en general. Blackwell publicó su texto sobre esgrima de espada corta para el populacho americano cuando se dio cuenta de que enseñar esgrima a unos pocos "raros" no era ni grato ni una carrera muy lucrativa en las colonias. Como afirma Blackwell,

Habiéndome encontrado, en mi reducida práctica en diversas partes de América, con muchas dificultades para introducir el ARTE de la Espada Corta, casi me desesperé, y ello es debido a la admiración que merece un arte tan ingenioso. 1734: A3

No sólo eran escasos los estudiantes adinerados, sino que aparentemente un segmento del público estadounidense sentía que la esgrima de las clases altas tenía poca utilidad y era, posiblemente, socialmente disruptiva respecto a una ciudadanía igualitaria. En un intento por popularizar su estilo de combate, Blackwell respondió esbozando una argumentación de seis puntos a favor de la esgrima, finalizando con la afirmación,

Aunque un hombre nunca vaya a luchar con su espada, no hay ningún ejercicio más saludable y placentero para el aprendiz que esta esgrima: trabajando todas las partes del cuerpo, fortalece las piernas, abre el pecho, surte de aire limpio, y de un comportamiento apuesto al cuerpo, un pisar majestuoso; y lo hace activo, vigoroso y vital; y también le permite servir a su amigo y a su país. 1734: ix

El público aparentemente respondió de un modo positivo a los argumentos de Blackwell, puesto que diversos maestros establecieron escuelas de esgrima en muchas ciudades litorales durante la era colonial.

Durante el s. XIX se habían desarrollado sistemas de combate estadounidenses únicos, y era común el cultivo de la marcialidad como medida de la masculinidad. Algunos de estos sistemas de combate americanos, como el combate con bastón con reglas americanas, estaban basados en modelos del Viejo Mundo. Otros, sin embargo, eran más enteramente estadounidenses. Gorn relata que en el sur estadounidense anterior a la guerra, donde la pelea era algo común, predominaba el "escoplear" o pelear con la intención de sacar un ojo al oponente como símbolo de victoria. Para distinguirse ellos mismos de los boxeadores y de los luchadores, los combatientes del sur etiquetaban intencionalmente a su estilo de combate como "violento" o "escopleado". "Escoplear" llegó a ser una práctica que estuvo tan extendida y aceptada que desarrolló su propio folklore y mitología popular (Gorn, 1985: 20-28). En otras partes de la nación, y entre otras clases, se aplicaban diferentes reglas de combate. En los "bosques del norte", por ejemplo, "dar pisotones" o derribar al oponente hasta que era posible atacarle con las botas de tachuelas, era con mucho más frecuente y socialmente aceptable que "escoplear". En el medio oeste, la lucha fue mucho más frecuente que otras formas de recreación combativa, llevando al desarrollo del estilo Catch-as-Catch-Can popularizado por los éxitos de los luchadores del medio oeste tales como el pre-presidencial Abraham Lincoln (vencedor en un combate con el campeón estatal de Louisiana en New Salem, Louisiana, en 1831) y Martin "Granjero" Burns (1861-1937), una de las primeras personas en hacer que fuese viable comercialmente la enseñanza de la lucha por correspondencia en la primera parte del s. XX. A lo largo de los s. XVIII y XIX, mientras la identidad estadounidense estaba ligada a la idea de la frontera americana, la afirmación de que "los primeros pobladores de la

Forjando hombres sobre el tapiz... $\diamond$ Geoffrey Wingard 
frontera eran los mejores luchadores" llegó a ser una profecía casi autorrealizada (aunque es importante advertir que los combates de lucha y otras demostraciones de artes viriles también tenían lugar en municipios y universidades) (Holliman, 1975: 149).

La élite estadounidense continuó patrocinando y participando en las artes viriles. La esgrima de bastón, un método de esgrima con espada de madera, era practicada por los cadetes de casi cualquier academia militar secundaria y post-secundaria del país durante el s. XIX. Theodore Roosevelt, campeón de la vida extenuante, apoyó la práctica de las artes viriles para todos los jóvenes y hombres americanos. Como presidente, Roosevelt recibió la visita de instructores estadounidenses y japoneses de lucha, boxeo, judo y combate con bastón, y practicó con ellos en la Casa Blanca. Roosevelt alentaba la práctica de las artes viriles tradicionales al igual que la de los nuevos y más modernos deportes ${ }^{3}$.

Sobre finales del s. XIX, sin embargo, la percepción de los estadounidenses de las artes viriles había empezado a cambiar. A pesar de que el cultivo de la masculinidad y de la fuerza era aún admirado, la práctica de las artes de combate tradicionales había empezado a declinar. Una razón de ello tenía que ver con las restricciones impuestas a los combates en áreas urbanas. A medida que América se convertía en una nación urbana, los excesos en el comportamiento, las excentricidades y la violencia que se permitía anteriormente en las comunidades rurales, y que era aceptada entre grupos de trabajadores masculinos tales como tripulantes de barcos fluviales, cuadrillas de leñadores, mineros, e incluso que estaba permitida en pequeñas comunidades étnicas urbanas caracterizadas por una fuerte solidaridad social, fue restringida. En las áreas urbanas, la gente de clase pobre y trabajadora se enfrentaba a una cultura de la élite, a prácticas religiosas, y a expectativas comerciales que diferían significativamente de su experiencia previa. Incapaces de competir materialmente con las élites, utilizaron el comportamiento social incluyendo la vestimenta, la etiqueta y la reputación para normalizar las relaciones con los administradores, propietarios y funcionarios urbanos. Se desalentaron las prácticas que ponían de manifiesto diferencias regionales y de clase, tales como la participación en combates de "escoplear" o "dar pisotones". Más aún, en las ciudades con sistemas judiciales modernos y fuerzas policiales, el recurso a la violencia personal para solventar agravios estaba severamente restringido. El editor de la revista online Journal of Manly Arts, Tony Wolf, (2000: 1), explica

Este periodo [la primera mitad del s. XIX] vivió la decadencia de la esgrima militar, del tiro con arco, etc., concomitante con los inexorables avances de las armas de fuego y de los explosivos. Las antiguas tradiciones del duelo de honor también declinaron, y los duelos fueron prohibidos finalmente en la mayoría de países "civilizados". Hacia finales del periodo, muchas naciones habían establecido fuerzas policiales profesionales, relevando teóricamente a la ciudadanía de la necesidad de llevar armas abiertamente.

Otras opiniones también afectaron a la práctica y prevalencia de las artes viriles en América. Las nuevas teorías sobre la higiene y la enfermedad exacerbaron la decadencia de la práctica de las recreaciones violentas. El contacto físico llegó a ser visto como un vehículo para la transmisión de la enfermedad. El contacto con los fluidos corporales, tales como la sangre y el sudor, en el contexto de la recreación, era particularmente desagradable para muchos estadounidenses de la élite en la era victoriana. El patrocinio y participación de la élite en muchas de las artes viriles declinó.

La esgrima fue la única de las artes viriles arquetípicas que siguió siendo patrocinada por las élites en gran medida. Esto fue posiblemente debido a la asociación de la esgrima con un ideal mítico anglosajón y debido a que el equipamiento de la esgrima reafirmaba la primacía material y social de las élites (Jackson-Lears, 1981: 107-140). Aldo Nadi, un maestro de esgrima italiano que acreditaba el mantenimiento de los ideales marciales clásicos en la era deportiva moderna, ha descrito la esgrima como única entre todos los deportes de contacto, afirmando, "La esgrima es un deporte de contacto

Revista de Artes Marciales Asiáticas $\diamond$ Volumen 4 Número 2 (8-21) - 2009 
-un contacto del acero, no de los puños o de los cuerpos-" (Nadi, 1943: 13). En el mismo ensayo, Nadi compara la esgrima con el boxeo, concluyendo que la esgrima es física, intelectual y moralmente superior. A medida que la urbanización y los preceptos de la ley continuaron desalentando las recreaciones violentas en la temprana América industrial, los miembros socialmente sensibles de otras clases sociales siguieron el parecer de las élites y disminuyó la petición de sangrientos combates de boxeo, luchas con bastón y otros tipos de enfrentamientos.

Los nacionalistas estadounidenses miraban de reojo a cualquier forma de recreación que pareciese celebrar una herencia extranjera. Los manuales de esgrima, guías de la más cosmopolita de las artes viriles, fueron reescritos con el tiempo para sistematizar y americanizar los diversos estilos de esgrima europeos ${ }^{4}$. La participación en estilos de lucha y en sistemas de boxeo que pareciesen celebrar con demasiada intensidad la herencia personal como inmigrante fueron vistos como evidencia de que el practicante no era lo suficientemente americano. Incluso los estilos estadounidenses de combate, tales como la lucha Catch-as-Catch-Can, sufrieron como consecuencia de su carácter rural y regional y su afinidad técnica con las tradiciones de lucha anglo-gaélicas de Lancashire y Cornwall 5 .

De los diversos factores que se combinaron para crear una atmósfera inhóspita para la práctica de las artes viriles tradicionales, y favorable para la introducción de nuevos deportes marciales basados en las artes marciales asiáticas en la América de la era industrial, la creación del deporte moderno tuvo un gran peso. El deporte moderno y los ideales del deporte fueron diseminados de las clases altas y medias a las clases trabajadoras y pobres. Al mismo tiempo, la creciente urbanización y el consiguiente aumento del temor al crimen urbano crearon una reacción contra los "higienizados" deportes modernos que contribuyó a que los estadounidenses aceptasen rápidamente los métodos de combate asiáticos. Finalmente, el desencanto ampliamente generalizado con la gestión y práctica de los deportes de combate tradicionales distanció a los seguidores de la recreación marcial de las artes viriles tradicionales incluso aunque muchos aún tenían preferencia por juegos marciales, forzándolos a buscar nuevos puntos de encuentro en los que participar en la recreación marcial. Al mismo tiempo, un popular, anti-modernista y nostálgico anhelo por el (en gran parte mítico) pasado pre-industrial hizo a la sociedad estadounidense receptiva a la introducción de las artes marciales asiáticas, particularmente del jujitsu y del judo, que parecían prometer una especie de iniciación simbólica a un ethos guerrero universal. El examen de este conjunto interconectado de factores es la única forma de explicar por qué las artes marciales japonesas fueron introducidas, comercializadas y rápidamente aceptadas en la sociedad americana.

A medida que se desarrollaba la ética del deporte moderno, primero entre las élites y entre los reformadores cristianos, y después entre los participantes de clases medias y trabajadoras, las actitudes populares hacia los deportes experimentaron una transformación radical ${ }^{6}$. Los deportes se transformaron de eventos festivos, locales, y de participación, en juegos codificados, multi-locales y nacionales apoyados por instituciones jerárquicas y regulados a niveles por encima de aquellos que ocupaban la mayoría de practicantes y espectadores. Sobre principios del s. XX, los deportes modernos, como opuestos a los juegos y competiciones participativas, habían llegado a ser "el aspecto más universal de la cultura popular" (Miller et al., 2001: 1). Un resultado eventual de este cambio en la composición del deporte estadounidense y del crecimiento de la actividad atlética moderna fue el desarrollo de una extraña dicotomía entre los seguidores del movimiento atlético, que enfrentaban dos visiones del deporte. Por una parte, los deportes eran vistos como instituciones limitadas por reglas que condicionaban la participación y promocionaban la asistencia de espectadores (sembrando las semillas del profesionalismo y del mercantilismo). Por otra parte, los deportes eran (idealmente) practicados por sí mismos, con la idea de que la práctica diligente y la buena deportividad generarían unos comportamientos y atributos positivos entre los jugadores tanto dentro como fuera del campo.

Forjando hombres sobre el tapiz... $\diamond$ Geoffrey Wingard 
Fue fundamentalmente esta última afirmación, la que mantenía que los deportes eran buenos tanto para el alma como para el cuerpo, la que alentó a los proselitistas del deporte a lanzar lejos sus redes para abarcar juegos y jugadores fuera de su círculo de clase alta. El ascenso de la nueva ética deportiva (y su proselitista acompañamiento moral, el Cristianismo Muscular) fomentó un deseo misionero por difundir el mensaje del deporte más allá de sus originales fronteras de raza, clase y nación. Los misioneros tanto Cristianos como atléticos llevaron el mensaje del deporte a las esquinas más alejadas de un mundo que estaba industrializándose rápidamente y consecuentemente le pusieron en contacto con juegos y actitudes foráneas a la sociedad occidental de clase alta y media. Estos movimientos misioneros introdujeron inicialmente las artes marciales japonesas (especialmente una nueva arte marcial, "científica", llamada judo) a Occidente.

A finales del s. XIX se había hecho visible una crisis en el deporte estadounidense. Los deportes que aparentemente eran aceptables para las asociaciones modernistas atléticas y Cristianas (tales como la YMCA) a menudo tenían poco atractivo para las masas, que se habían criado con una dieta de deportes sangrientos. Aquellos que seguían los deportes modernos eran vistos como elitistas y afeminados por los partidarios de los deportes de combate. Al mismo tiempo, sin embargo, la justificación moral para participar en deportes de combate fue usurpada por los que se dedicaban a la ética del deporte. En consecuencia, aquellos que demandaban los deportes de combate según la tradición de las artes viriles estaban sujetos al ridículo por apoyar una anacrónica tradición antisocial. Prevaleció una posición inestable, en la cual los atletas modernos parecían afeminados a un segmento significativo del público por su rechazo a participar en la recreación marcial, mientras aquellos que participaban en las artes de combate eran caracterizados como deficientes moralmente. Algunos defensores de los deportes trabajaron diligentemente para resolver esta situación, diseñando un deporte que pudiese satisfacer los criterios morales modernos a la vez que fuese atractivo para las motivaciones tradicionales de base, pero no era un tema fácil de resolver. Tan tarde como en 1946, cuando el campeón de boxeo británico Bruce Woodcock fue derribado por el americano Tami Mauriello, el comentarista deportivo Red Smith escribió, "[Woodcock combatió] como alguien que aprendió boxeo de un libro y aún creyese que es un arte viril" (Smith, 1996: 61).

A finales del s. XIX y principios del XX, la participación en baloncesto, atletismo y ciclismo prosperó, pero el patrocinio de esos deportes por parte de los espectadores fue débil. Al mismo tiempo, el boxeo a puño limpio tradicional estaba, cada vez más, bajo la jurisdicción de la censura legal y la lucha estaba empezando a mostrar signos de ser más un espectáculo que una competición. Sin embargo, el público continuó patrocinando las competiciones marciales locales (en ocasiones ilegales). Claramente, las artes viriles aún tenían una cierta resonancia para el populacho americano. Sin embargo, no iban a recibir el patrocinio o el apoyo del que disfrutaban los deportes más higiénicos.

\section{Las Artes Marciales Asiáticas ocupan su lugar en los EE.UU.}

Los preconizadores del deporte buscaron por todo el mundo una actividad que pudiese cumplir un nuevo conjunto de criterios. Sentían que necesitaban una actividad que tuviese el atractivo de las artes viriles tradicionales, pero que estuviese libre de la sórdida historia del boxeo, libre de la caricatura rural en que se había convertido la lucha, y libre de las fronteras elitistas de clase de la esgrima. También necesitaban una actividad que fuese moderna en su aproximación, una que encarnase las características del deporte moderno tales como el mantenimiento regular del récord, reglas estandarizadas, requisitos respecto al equipamiento, y normas de la era industrial (Guttmann, 1978: 16). Finalmente, el deporte ideal tenía que ser atractivo no sólo a los entusiastas del deporte, sino también a la población en general. Eso quería decir que la actividad tenía que responder a alguna necesidad percibida como pública tal como el mantenimiento de la salud, la mejora de la imagen física o, en relación a cualquier discusión sobre las artes viriles, la necesidad de autodefensa. En la periferia del mundo occidental industrializado,

Revista de Artes Marciales Asiáticas $\diamond$ Volumen 4 Número 2 (8-21) - 2009 
estos reformadores del deporte descubrieron un deporte que satisfacía estos criterios. Descubrieron el estilo japonés de lucha cuerpo a cuerpo llamado judo.

El judo era una síntesis moderna de sistemas japoneses de combate sin armas (jujutsu) creado en 1882 por el especialista en educación física japonés y experto en jujutsu Kano Jigoro. Kano había estudiado estilos clásicos de jujutsu pero había considerado que eran inadecuados para el temperamento del Japón moderno y poco prácticos para el estudio moderno? ${ }^{7}$ Kano, un educador profesional, refinó posteriormente las artes de los antiguos guerreros y organizó una nueva forma sistemática de enseñanza de las habilidades de los antiguos samuráis. Kano basó su nuevo sistema en dos premisas: que la práctica del deporte tenía que ser segura para los participantes (a diferencia de los antiguos estilos de jujutsu, en los cuales los practicantes se lesionaban a menudo) y que el deporte tenía que ser atractivo a los practicantes de cualquier nivel de habilidad y clase social. Para incrementar el atractivo del judo tanto para la sociedad racional industrial como para los círculos conservadores anti-modernistas, Kano buscó integrar las teorías modernas sobre el entrenamiento y la competición (influenciadas por el contacto japonés con Occidente) con filosofías guerreras neotradicionales. De acuerdo con Donn Draeger y Robert Smith, "El judo se volvió hacia la educación física y la cultura" (1980: 139). Incluso Kano, conscientemente, planeó el nombre de su nueva arte marcial para reflejar las características físicas y morales que consideraba que podrían popularizarla tanto como deporte moderno y como arte viril. Llamó formalmente a su nuevo sistema Nippon Den Kodokan Judo, "una expresión que significa el mejor budo de Japón"” (Draeger, 1996: 118). Debe advertirse, sin embargo, que no todos los cambios iniciados por Kano y sus contemporáneos conocieron un éxito sin mácula. En Modern Bujutsu and Budo: The Martial Ways of Japan, una de las primeras revisiones rigurosas sobre las artes marciales japonesas en inglés, el autor es crítico respecto al judo y sus derivados afirmando que "Los sistemas de lucha cuerpo a cuerpo son los descendientes de las series de tácticas politípicas que tuvieron sus inicios en los estilos marcialmente inefectivos del jujutsu clásico del último período Edo" (Draeger, 1996: 60). Otras artes marciales, fundamentalmente diversos estilos de kárate, también han sido criticados por su moderno énfasis en la competición y estandarización de la práctica8 .

Desde su nacimiento, el judo satisfacía los criterios que buscaban los defensores occidentales del deporte. El judo también satisfacía la mayoría de las siete características que el historiador Allen Guttmann ha determinado que deben estar presentes para que una actividad sea considerada como un deporte moderno. Estas características comprenden el tipo de cambios que los reformadores del deporte habían hecho a los deportes occidentales del s. XIX, tales como diversos tipos de fútbol y carreras de bicicleta, características que también eran evidentes en el judo. Los criterios de Guttmann incluyen el secularismo, la igualdad de oportunidades, la especialización de roles, la racionalización, la organización burocrática, la cuantificación, y la búsqueda de récords (Guttmann, 1978: 16). El judo ha sido examinado bajo los parámetros de Guttmann y se comprobó que satisfacía la mayoría de estas condiciones. Carr determinó que el judo sólo fracasa al ser calificado como deporte moderno en su relativa incapacidad para ser "cuantificado". Esto, sin embargo, es una condición que comparte con muchos deportes orientados a la ejecución, tales como el patinaje artístico, la gimnasia y la danza competitiva, en los que existen unos estándares y un juicio subjetivo, y no por ello deben descalificarse automáticamente (Carr, 1993: 185-187).

Además de ajustarse firmemente al molde del deporte moderno, el judo tenía una utilidad obvia para los estadounidenses urbanos. Era un sistema de autodefensa que, al menos teóricamente, no requería que el actor tuviese una masa o una fuerza aplastante para doblegar a un oponente. Estaba formado por un buen número de técnicas aplicables en un amplio abanico de circunstancias y podía enriquecerse con los métodos de combate occidentales tanto como fuese necesario. Su práctica era supuestamente segura para hombres, mujeres y niños, y desde un principio se ofreció instrucción en judo en Inglaterra y en los Estados Unidos tanto a hombres como mujeres ${ }^{9}$. El judo podía ser también practi-

Forjando hombres sobre el tapiz... $\diamond$ Geoffrey Wingard 
cado fácilmente en el limitado espacio disponible en las abarrotadas ciudades industriales (Matsudaira, 1910: 117). Finalmente, el judo era intencionalmente un deporte filosófico y moral (Lindsay \& Kano, 1889: 204-205; Carr, 1993: 168). Kano Jigoro incluyó conscientemente la educación de preceptos morales como parte del currículum del judo. Derivada de la filosofía tradicional japonesa y del código del guerrero japonés, el bushido, la filosofía del judo contenía elementos que eran directamente atractivos a los entusiastas de los aspectos morales del deporte.

Aquellos que abogaban por la expansión de las oportunidades deportivas, sobre la base de que éstas contribuían al desarrollo moral a través de la ética de la buena deportividad y del juego limpio, observaron que el respeto por el rival y el autocontrol eran piedras angulares de la práctica del judo. Como advirtió un posterior observador, los entusiastas de los deportes del s. XIX creían que "En las artes marciales de Asia el conflicto parece muy severo, aunque la consideración del oponente es muy alta" (Luschen, 1981: 201). Incluso muchos anti-modernistas occidentales, que eran en el mejor de los casos escépticos respecto al movimiento deportivo moderno, aceptaron a regañadientes el judo ya que dibujaban un paralelismo entre el antiguo código feudal samurái (bushido), sobre el que se basaba parcialmente la filosofía del judo, y la legendaria hidalguía de los caballeros errantes ingleses ${ }^{10}$.

En Japón, el judo fue considerado uno de los caminos marciales de la nueva era (shin budo). Estas artes eran vistas como distintas y superiores a los meros sistemas de combate, puesto que tenían un componente moral explícito. El filósofo japonés del s. XIX Aizawa Yasushi (1781-1863) afirmaba, "Conocer la etiqueta y el honor, preservar el camino del caballero, esforzarse por la austeridad, y por tanto ser un baluarte del estado, es budo" (Friday, 1997: 7). Aunque era innegablemente extranjero para los proselitistas de los deportes occidentales, el judo parecía referirse a un sentimiento guerrero universal, una idea que tuvo un atractivo cada vez mayor entre los estadounidenses expansionistas. Más aún, los códigos morales del judo y del bushido tenían al menos ciertas similitudes con la ética postulada por los defensores del movimiento deportivo moderno. En el comentario a una conferencia realizada en la Japan Society en 1910, el Conde Mutsu, un miembro del gobierno Meiji y de la British Japanese Society, señaló, "Nuestro Bushido es vuestra deportividad" (Matsudaira, 1910: 133). En el Bushido de Nitobe, la guía en lengua inglesa de 1905 a la cultura japonesa a través de la filosofía de su tradición guerrera, los capítulos tres a nueve se titulan:

\author{
III. Rectitud o Justicia \\ IV. Valor, Espíritu de Audacia y de Resistencia \\ $\checkmark$. Benevolencia, Bondad para con el dolor \\ VI. Cortesía \\ VII. Veracidad y Sinceridad \\ VIII. Honor \\ IX. El deber de Lealtad
}

Estos títulos de capítulo tienen notables similitudes con las metas adoptadas por los organizadores de clase dirigente de los deportes modernos, los cuales buscaban inculcar las virtudes de coraje, honor, lealtad, buena deportividad, y caridad cristiana en jugadores y espectadores. Los primeros entusiastas de judo muy probablemente habrían estado de acuerdo con el comentario de Yuasa Yasuo (1925-2005) quien señalaba: "El entrena-

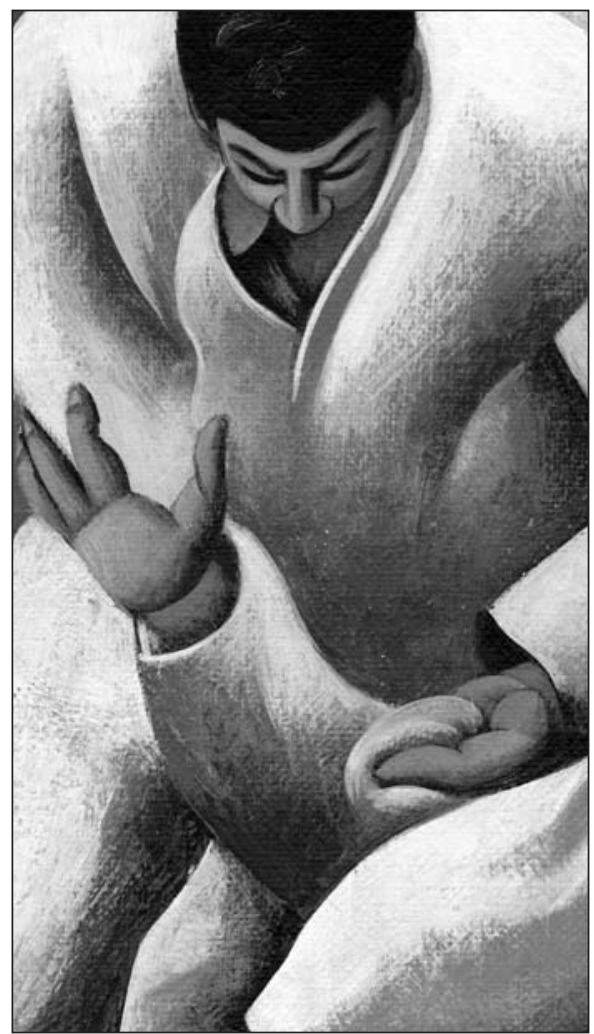

Detalle de la pintura de Curtis Parker. 


\section{Los historiadores de la cultura, asi como los historiadores}

de las artes marciales $y$ del deporte, pueden aprender una lección de la historia a través de los cambios en la práctica de las artes viriles en América.

miento en los deportes tiene como objetivo desarrollar la capacidad del cuerpo... Por otra parte, la meta original del camino del bushi [guerrero] es desarrollar la capacidad mental (o espiritual)" (1993:32).

Aunque nunca fue lo suficientemente popular como para rivalizar con los deportes "americanos" como el fútbol americano, el béisbol o incluso el boxeo profesional (con guantes), resucitado a principios del s. XX, el judo estableció el escenario para la introducción de otros deportes marciales en América. A partir de principios del s. XX, "olas" sucesivas de inmigración de diversos deportes de combate de todo el mundo llegaron a ser casi instantáneamente populares sólo para desaparecer de la conciencia del público americano casi tan rápidamente. Desde los años 50, los deportes marciales del este de Asia, con nombres esotéricos tales como Wing Chun, Kempo (Kenpo), Ninjutsu, Muay Thai, y el sincrético arte marcial llamado Brazilian jujitsu, han logrado sucesivamente la popularidad y el éxito comercial en el mercado estadounidense de los deportes marciales. Los sistemas de combate y los "maestros" estadounidenses se beneficiaron de estas olas de popularidad, incluso mientras vendían sus propios sistemas de artes marciales como un arma para desbaratar los "trucos" nuevos o extranjeros (Burns, 1913).

Este proceso de adquisición, comercialización y diseminación, que empezó tempranamente en la historia americana con la esgrima y con los estilos de combate estadounidenses, es característico de las diversas interacciones culturales americanas. Más aún, se orienta a una situación en la que "[nosotros] nos encontramos perplejos mientras intentamos equilibrar la victoria con el juego limpio, la agresividad con el control, la libertad con la técnica, y lo individual con lo comunitario" (Hardy, 1990: 77).

Forjando hombres sobre el tapiz... $\diamond$ Geoffrey Wingard 
${ }^{3}$ La descripción más cabal de las actividades marciales de Roosevelt se encuentra en Donovan (1909). Para un análisis más completo de las implicaciones de la participación de Roosevelt en deportes marciales sobre su propia persona y sobre las artes en América, véase Burdick (1999: 22-54).

${ }^{4}$ Véase Cass (1930: 17-18), como ejemplo de un manual de esgrima conscientemente americanizado.

5 Puede verse un ejemplo de la continua diseminación y adaptación de la lucha anglogaélica durante el s. XX en los Estados Unidos en Pittman (1999: 48-57), específicamente pp. $49 \& 57$.

${ }^{6}$ El ascenso de los deportes modernos ha sido tratado en algunos trabajos con gran detalle, entre ellos los de Guttmann (1978) y Holt (1989).

7 En 1868, el emperador Meiji arrebató el control del gobierno de Japón al último shogun Tokugawa. Para afianzar su posición y sostener el control sobre los samuráis conservadores, el emperador emprendió una carrera para modernizar Japón rápidamente. Se prohibieron muchos aspectos del antiguo régimen y otros cayeron pronto en desuso. Algunas artes marciales cambiaron su currículum para ser atractivas a audiencias más populares. Una discusión arquetípica del carácter simbólico y de las implicaciones de estos cambios en la cultura marcial japonesa se incluye en las memorias de Funakoshi Gichin (1975: 1-7).

${ }^{8}$ Con una frecuencia cada vez mayor, recientemente se han publicado análisis en el ámbito académico sobre los primeros años del judo, incluyendo Carr (1993: 167-188), Smith (1996: 60-65), y Bowen (1999: 43-53).

9 Barton-Wright (1902: 261-264) y Norman (1905) exaltaron la idoneidad del judo y ejercicios similares independientemente de las barreras de clase, raza y género.

${ }^{10}$ Es importante advertir que el ideal guerrero japonés que admiraban los ingleses fue un concepto derivado en gran medida de Nitobe (1905) y de los panfletos publicados por occidentales que residieron durante breves periodos en Japón (e.g. Norman, 1905: 1-3). Nitobe había sido educado en escuelas privadas inglesas y era cristiano. Es muy probable que su versión del código del honor samurái estuviese altamente idealizada, si no adaptada específicamente para atraer a la audiencia inglesa. De un modo similar, los registros de los panfletistas también deben ser vistos críticamente, puesto que sus motivos eran frecuentemente comerciales o evangélicos.

\section{BIBLIOGRAFÍA}

Barton-Wright, E.W. (1902). Ju-jitsu and Ju-do. Transactions and proceedings of the Japan Society, London 5: 261-264.

BlACKWELL, E. (1734). A compleat system of fencing: Or, the art of defence. Williamsburg: William Parks.

Bowen, R. (1999). Origins of the British Judo Association, the European Judo Union \& the International Judo Federation. Journal of Asian Martial Arts, 8(3): 43-53.

Burdick, D. (1999). The American way of fighting: Unarmed defense in the United States, 1845-1945. Ph.D. Dissertation, University of Indiana.

Burns, M. (1913). Jiu Jitsu-Self defense and their relation to wrestling: Lesson XII (Book VI). Omaha: Farmer Burns School of Wrestling.

CARR, K. (1993). Making way: War, philosophy and sport in Japanese judo. Journal of Sport History, 20(2): 167-188.

CAss, E. (1930). The book of fencing. Boston: Lothrop, Lee and Shepard, Co.

Donovan, M. (1909). The Roosevelt that I know: Ten years of boxing with the president - and other memories of famous men. New York: B.W. Dodge and Co.

Draeger, D. (1996). Modern bujutsu and budo: The martial arts and ways of Japan. New York: Weatherhill.

Forjando hombres sobre el tapiz... $\diamond$ Geoffrey Wingard 
Draeger, D. \& R. Smith. (1980). Comprehensive Asian fighting arts. New York: Kodansha International.

Dykhuizen, J. (2000): Culture, training and perception of the martial arts: Aikido's example. Journal of Asian Martial Arts, 9(3): 9-31.

FRIDAY, K. (1997). Legacies of the sword: The Kashima-Shinryu and samurai martial culture. Honolulu: University of Hawai'i Press.

FunAKOSHI, G. (1975). Karate-do, my way of life. New York: Kodansha International.

GORN, E. (1985). Gouge and bite, pull hair and scratch: The social significance of fighting in the southern backcountry. American Historical Quarterly, 90(1): 18-43.

Gray, W. Russell. (1987). For whom the bell tolled: The decline of British prize fighting in the Victorian era. Journal of Popular Culture, 21(2): 53-64.

Guttmann, A. (1978). From ritual to record: The nature of modern sports. New York: Columbia University Press.

Holliman, J. (1975). American sports 1785-1835. No. 34 of Perspectives in American History. Philadelphia: Porcupine Press.

Holt, R. (1989). Sport and the British: A modern history. Oxford: Clarendon Press.

HARDY, S. (1990). Entrepreneurs, structures and sportgeist. In Essays on Sport History and Sport Mythology, edited by Donald Kyle and Gary Stark. College Station: Texas A\&M University Press.

JaCKSON-LeARs, T. (1981). No place of grace: Anti-modernism and the transformation of American culture 1880-1920. New York: Pantheon Books.

Jones, H. (1943). Judo, jiu-jitsu, and hand-to-hand fighting: A list of references. Washington, D.C.: The Library of Congress Division of Bibliography.

LiNDSAY, T. \& J. KANO (1889). Jiujutsu the old samurai art of fighting without weapons, Transactions of the Asiatic Society of Japan, 16: 192-205.

Luschen, G. (1981). The system of sport-Problems of methodology, conflict and social stratification. In Handbook of the Social Science of Sport, edited by Gunther Luschen and George Sage. Champaign, IL: Stipes Publishing Co.

Mandell, R. (1984). Sport: A cultural history. New York: Columbia University Press.

MatsudairA, T. (1910). Sports and physical training in modern Japan. Transactions and proceedings of the Japan Society, London, 8: 114-134.

Miller, T., G. Lawrence, J. McCay \& D. Rowe. (2001). Globalization and sport: Playing the world. London: Sage Publications.

Nadi, A. (1943). On fencing. New York: G.P Putnam's Son.

Nitobe, I. (1905). Bushido. New York: G.P. Putnam's Sons.

Norman, F. (1905). The righting man of Japan, the training and exercises of the samurai. London: Archibald Constable \& Co. Ltd.

Pittman, A. (1999). Combat wrestling: Geoghan's blend from East and West. Journal of Asian Martial Arts, 8(4): 48-57.

SAnsone, D. (1988). Greek athletics and the genesis of sport. Berkeley: University of California Press.

Skidmore, M. (1995). Oriental contributions to Western popular culture: The martial arts. Journal of Popular Culture, 25(1): 129-148.

Smith, R. (1996). The masters contest of 1926: An epiphany in judo history. Journal of Asian Martial Arts, 5(3): 60-65.

WingaRD, G. (2003). Sport, industrialism and the Japanese gentle way: Judo in late Victorian England. Journal of Asian Martial Arts, 12(2): 16-25.

Wolf, T. (2000). An introduction to the Journal of Manly Arts. Electronic Journals of Martial Arts and Sciences: Journal of Manly Arts, http://ejmas.com/jmanly/jmanlymission.htm (17 de febrero de 2003).

Yuasa, Y. (1993). The body, self-cultivation, and ki energy. New York: State University of New York Press.

Revista de Artes Marciales Asiáticas $\diamond$ Volumen 4 Número 2 (8-21) - 2009 